\title{
ELETROMIOGRAFIA DO BÍCEPS BRAQUIAL EM CONTRAÇÕES DINÂMICAS
}

\author{
ELECTROMYOGRAPHY OF THE BICEPS BRACHII DURING DYNAMIC CONTRACTIONS
}

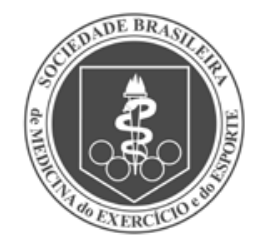

Relato de Caso

\author{
ELECTROMIOGRAFÍA DEL BÍCEPS BRAQUIAL EN CONTRACCIONES DINÁMICAS
}

\begin{abstract}
Lucenildo Silva Cerqueira
(Educador Físico) ${ }^{1}$

Joyce Ferreira Carvalho

(Educador Físico) $)^{1,2}$

Fernando Augusto Monteiro

Sabóia Pompeu (Educador Físico) ${ }^{1}$

1. Laboratório de Biometria -

LADEBIO - PPGEF/ UFRJ.

2. Laboratório de Bioquímica

Aplicada ao Desempenho Motor

- LABIDEM - IEFD/ UERJ.
\end{abstract}

\section{Correspondência:}

Fernando A.M.S. Pompeu

Programa de Pós-Graduação em

Educação Física - EEFD/ UFRJ.

Av. Carlos Chagas Filho, 540,

Cidade Universitária. 21941-599 -

Rio de Janeiro, RJ, Brasil.

ladebio@eefd.ufrj.br

\section{RESUMO}

Introdução: A reprodutibilidade da atividade eletromiográfica de superfície (EMG) é mais estudada em protocolos que envolvem contrações isométricas. Alguns fatores relacionados às contrações dinâmicas podem contribuir para a instabilidade do sinal mioelétrico e dificultar a reprodutibilidade da medida da EMG. Objetivo: Determinar a reprodutibilidade teste-reteste e quantificar o erro técnico da medida da frequência mediana (FM) e da amplitude (root mean square, RMS) da EMG, em contrações dinâmicas e estáticas. Métodos: Dez sujeitos foram testados em dois dias, sendo a atividade EMG registrada na maior porção do bíceps braquial. No primeiro dia foi feito o teste de uma repetição máxima (1RM) e de contração voluntária máxima (CVM). No segundo dia foram realizadas duas séries de 10 contrações dos flexores do cotovelo com 75\% de 1RM, entremeadas por $1 \mathrm{~h}$ em repouso. Antes de cada série foi realizada uma CVM. Resultados: Para a FM e RMS foi observado alto coeficiente de correlação intraclasse para o sinal do bíceps braquial $(\mathrm{CCl}=0,90-0,98$ para a $\mathrm{FM} ; \mathrm{CCl}=$ 0,89-0,94 para o RMS) e de baixo para moderado coeficiente de variação (CV = 2,5-6,2\% para a FM; CV $=14,6-16,3 \%$ para o RMS) em ambos os testes. Não foi observada diferença significativa entre teste e reteste $(p>0,05)$. Conclusão: A FM e o RMS apresentam alta confiabilidade e baixo a moderado erro em contrações estáticas e dinâmicas, possibilitando o uso da EMG para investigação da fadiga e de desordens neuromusculares.

Descritores: músculo esquelético, fadiga muscular, análise espectral, eletromiografia.

\section{ABSTRACT}

Introduction: Reproducibility of electromyographic activity (EMG) is the most studied in protocols involving isometric contractions. Some factors related to dynamic contractions may contribute to the instability of the myoelectric signal and affect the reproducibility of the measurement of EMG. Objective: To determine the test-retest reproducibility and quantify the technical error of the measurement of the median frequency (MF) and amplitude (root mean square, RMS) of the surface EMG in static and dynamic contractions. Methods: Ten subjects were tested in two days with the EMG activity recorded for the greatest portion of the biceps brachii tendon. In the first day the subjects did one test of maximum repetition (1MR) and maximal voluntary contraction (MVC). On the second day the subjects performed two sets of 10 dynamic contractions of the elbow flexors at 75\% of 1 MR interspersed by $1 \mathrm{~h}$ at rest. Before each set one MVC was performed. Results: For both MF and RMS, was observed high intraclass reliability for biceps brachii (ICC $=0.90-0.98$ for MF; ICC $=0.89-0.94$ for RMS) and low to moderate coefficient of variation (CV $=2.5-6.2 \%$ for MF; $C V=14.6-16.3 \%$ for RMS) in both tests. No significant difference between test and retest was found ( $p>0.05$ ). Conclusion: The MF and RMS have high reliability and low to moderate error in static and dynamic contractions, enabling the use of EMG for the investigation of fatigue and neuromuscular disorders.

Keywords: skeletal muscle, muscle fatigue, spectrum analysis, electromyography.

\section{RESUMEM}

Introducción: La reproducibilidad de la actividad electromiográfica de superficie (EMG) es más estudiada en protocolos que involucran contracciones isométricas. Algunos factores relacionados con las contracciones dinámicas pueden contribuir para la inestabilidad de la señal mioeléctrica y dificultar la reproducibilidad de la medida de la EMG. Objetivo: Determinar la reproducibilidad prueba-nueva prueba y cuantificar el error técnico cuanto a la medida de la frecuencia mediana (FM) y de la amplitud (root mean square, RMS) de la EMG de superficie, en contracciones dinámicas y estáticas. Métodos: diez individuos fueron examinados en dos días, siendo la actividad EMG registrada en el área más grande del bíceps braquial. En el primer día, se hizo la prueba de una repetición máxima (1RM) y de contracción voluntaria máxima (CVM). En el segundo día, se realizaron dos series de 10 contracciones de los flexores del codo, con $75 \%$ de IRM, alternadas por 1 hora en reposo. Antes de cada serie, se hizo una CVM. Resultados: Para la FM y el RMS se observó un alto coeficiente de correlación para la señal del bíceps braquial (CCl =0,90-0,98 para la $F M ; C C l=0,89-0,94$ para el RMS) y un coeficiente de variación de bajo para moderado (CV =2,5-6,2\% para 
la FM; CV = 14,6-16,3\% para el RMS) en ambas pruebas. No se observó una diferencia significativa entre prueba y nueva prueba $(p>0,05)$. Conclusión: La FM y el RMS presentan alta confiabilidad, y error de bajo a moderado en contracciones estáticas y dinámicas, posibilitando el uso de la EMG para investigación de la fatiga y de desórdenes neuromusculares.

Palabras clave: músculo esquelético, fatiga muscular, análisis espectral, electromiografía.

Artigo recebido em 07/12/2010, aprovado em 22/10/2013

\section{INTRODUÇÃO}

A fadiga associada a mudança no desempenho pode gerar alteração no controle do movimento ${ }^{1-3}$ e aumentar os riscos de lesões musculares ${ }^{4-6}$. A eletromiografia de superfície (EMG) é amplamente usada para investigação dos mecanismos envolvidos na fadiga neuromuscular e declínio da força muscular ${ }^{7,8}$. Este exame também pode fornecer preciosas informações para o tratamento de disfunções neuromusculares ${ }^{5,6}$. O sinal EMG reflete a somação linear algébrica dos sinais elétricos gerados pelas unidades motoras (UM) ativas dentro da área de registro do eletrodo ${ }^{9}$. A amplitude e a frequência do sinal EMG podem refletir a ativação e a velocidade de condução do potencial de ação nas UMs ${ }^{10,11}$, quantificados, respectivamente, pelo valor eficaz (root mean square - RMS) e frequência mediana (FM).

A fadiga relacionada ao decréscimo da ativação muscular voluntária tem sido estudada tipicamente através de mensurações do sinal EMG durante contrações estáticas voluntarias ${ }^{6,7,12}$, antes e após exercícios isocinéticos repetidos ${ }^{13}$, em exercícios isocinéticos ${ }^{7,14}$ e em exercícios dinâmicos ${ }^{3,15}$. O entendimento dos mecanismos envolvidos na fadiga neuromuscular em atividades ocupacionais e dinâmicas, entretanto, parece ser mais relevante para as funções da vida diária ${ }^{3,16}$. Exercícios funcionais são usados por profissionais da saúde, como fisioterapeutas que pretendem reabilitar pacientes lesionados ${ }^{5-6}$, ou personal trainers que querem induzir a hipertrofia muscular de um indivíduo saudável3,16,17.

Durante contrações dinâmicas diversos fatores como mudanças no número de unidades motoras ativadas, na relação força/potência ao longo do movimento, no comprimento do músculo ${ }^{18,19}$ podem levar a interpretações erradas da EMG ${ }^{20}$. Outros fatores como mudanças na velocidade de condução da fibra muscular devidas à fadiga ${ }^{18,19}$, podem agravar a não estacionaridade do sinal mioelétrico e dificultar a reprodutibilidade da medida da EMG.

O conhecimento da reprodutibilidade e do erro típico ${ }^{21}$ associado à medida da atividade EMG é crucial para a interpretação dos resultados das investigações e esta tem sido estudada principalmente em ensaios envolvendo contrações isométricas ${ }^{14,22}$, usando equipamento isocinético de alto custo ${ }^{22}$, porém, pouco investigada em tarefas dinâmicas, mais próximas de atividades cotidianas do dia a dia. Para mensurações da reprodutibilidade, o coeficiente de correlação intraclasse (CCl) é preferível ao coeficiente de correlação de Pearson (r). O último não é sensível a fatores como mudanças na média e no desvio padrão que tendem a superestimar a reprodutibilidade ${ }^{14,22}$.

O entendimento do comportamento neuromuscular e dos erros associados a esta medida, em atividades ocupacionais e dinâmicas, possibilita o desenvolvimento de melhores métodos de avaliação e tratamento das disfunções neuromusculares ${ }^{5,6}$. Essa é a principal justificativa para realização do presente estudo. Com base nos estudos supracitados, levantou-se a hipótese de que o exercício submáximo promove a fadiga do bíceps braquial, cabeça longa, mas não compromete a reprodutibilidade da EMG em sujeitos jovens saudáveis, sendo a fadiga muscular constatada pela alteração do espectro de potência do sinal EMG em direção às baixas frequências, diminuindo a FM e aumentando a RMS. $O$ objetivo deste estudo foi determinar a reprodutibilidade das medidas da
FM e RMS em exercícios com contrações sustentadas e dinâmicas, assim como quantificar o erro técnico da medida (s) associado a essas variáveis. Secundariamente, pretendeu-se verificar a viabilidade na aplicação do sinal EMG através de sua análise no domínio da frequência como parâmetro para determinação e diferenciação no comportamento da fadiga muscular localizada no músculo bíceps braquial.

\section{MÉTODOS}

Dez sujeitos (sendo duas mulheres) fisicamente ativos ( $22 \pm 2$ anos; $168,8 \pm 4$ cm, 68,3 \pm 8,1 kg) participaram deste estudo. Recomendou-se a manutenção da dieta mista nas $48 \mathrm{~h}$ precedentes ao exame. Solicitou-se a abstinência de alimentos e cafeína nas três horas prévias ao esforço. Cada sujeito foi informado quanto aos riscos associados aos procedimentos adotados. Um termo de esclarecimento e consentimento foi lido e assinado. Todos os procedimentos aqui adotados foram aprovados pelo Comitê de Ética Local para Experimentos com Seres Humanos (Rio de Janeiro, CEP/ HSE 000.021/99). Este estudo foi realizado conforme a Declaração de Helsinki.

A coleta dos dados foi realizada em duas visitas, separadas por um mínimo de 48 e máximo de 72 horas. Na primeira visita foram medidas a massa corporal, estatura e dobras cutâneas ${ }^{23}$. Após esse procedimento, o indivíduo foi orientado a realizar o teste de uma repetição máxima (1RM). Antes do teste de 1RM foi realizado um teste de contração voluntária máxima (CVM) com duração de oito segundos para obtenção do sinal EMG. No teste de 1RM, a carga foi inicialmente estimada e, em seguida, adicionada ou subtraída até que fosse atingida uma carga com a qual o sujeito conseguisse realizar somente 1RM. Essa carga foi estimada com base na vivência do avaliado e experiência do avaliador. O intervalo de descanso entre tentativas foi de cinco a seis minutos. Não mais do que cinco tentativas foram permitidas na sessão de familiarização (em média, três tentativas foram necessárias para 1RM). Os participantes foram verbalmente encorajados a desempenhar o máximo.

Na segunda visita os sujeitos realizaram duas séries de 10 contrações dinâmicas dos flexores do cotovelo, com a carga estimada em $75 \%$ de 1 RM. As séries foram entremeadas por uma hora em repouso. Antes de cada série de 10 contrações foi realizada uma CVM. Entre a CVM e o teste com 75\% de 1RM foram dados cinco minutos de repouso. Para todos os testes os sujeitos foram posicionados sentados, com joelhos e quadris flexionados em aproximadamente $90^{\circ}$, em um banco scot desenvolvido para este fim, com o cotovelo posicionado em aproximadamente $160^{\circ}$, e ombro abduzido em aproximadamente $70^{\circ}$. O ritmo de execução foi estabelecido por meio de um metrônomo com cadência em 30 batidas por minuto.

\section{Registro eletromiográfico}

O registro eletromiográfico foi feito em todos os testes. O sistema de aquisição foi composto por um conversor analógico-digital de 12 bits e faixa dinâmica de $\pm 5 \mathrm{~V}$ (NI-DAQmx 8.6, National Instruments, Austin, TX). Os sinais foram armazenados em um computador para posterior análise. Para captação dos sinais foi utilizado um sistema de cabos com amplificadores, com banda de frequência limitada entre 10 e $500 \mathrm{~Hz}$ e ganho total de 1.200 vezes conforme as normas da European Recommendations for 
Surface Electromyography ${ }^{24}$. O eletromiógrafo possui aterramento e aquisição simultânea para todos os canais. Dois eletrodos circulares $(20 \mathrm{~mm}$ diâmetro, 20 mm de distância intereletrodo) de superfície de Ag-AgCl (Meditrace ${ }^{\circledR} 200$ - KENDALL, Canadá) foram posicionados na cabeça longa do bíceps braquial de acordo com as recomendações da SENIAM ${ }^{24}$, após preparação da pele (tricotomia de pelos, abrasão e limpeza com álcool). A frequência de amostragem adotada foi de $1.200 \mathrm{~Hz}$.

\section{Processamento dos sinais da EMG}

Para o processamento dos sinais da EMG durante as contrações dinâmicas, os sinais EMG correspondentes a cada contração foram separados em janelas com duração de dois segundos. Para diminuir os problemas da não estacionaridade do sinal EMG ${ }^{12}$ sobre a análise espectral das contrações dinâmicas adotaram-se janelas de um segundo nas rotinas de processamento, a partir do início da contração marcada visualmente ${ }^{12}$. Os sinais de EMG da CVM também foram previamente separados em trechos com duração de dois segundos.

Os sinais EMG foram processados através de rotinas específicas desenvolvidas em ambiente MatLab ${ }^{\circledR}$ (MathWorks, EUA). Na primeira fase de processamento aplicou-se ao sinal EMG um filtro digital passa-banda tipo Butterworth de 10 a ordem e frequência de corte entre 20 e $500 \mathrm{~Hz}$. Os dados contidos nos períodos de um segundo foram aplicados à transformada discreta de Fourier para obtenção de suas respectivas densidades espectrais de potência e para o posterior cálculo da FM. A FM divide o espectro de potência em duas áreas iguais. A escolha da mediana deveu-se às propriedades estatísticas da medida, que não é sensível a valores extremos. Calculou-se o valor RMS em janelas de 50 milissegundos do trecho de um segundo da EMG de cada contração. Todos os valores RMS foram também normalizados pelo RMS obtido na CVM da primeira visita.

\section{Análise estatística}

O tratamento estatístico foi realizado através dos aplicativos Statistical Package for the Social Sciences ${ }^{\circledR}$ (SPSS, EUA). Empregou-se a estatística descritiva com a média \pm erro padrão da média (EPM). O grau de associação entre os testes foi determinado através do coeficiente de correlação intraclasse $(\mathrm{CCl})$. O algoritmo $\mathrm{CCl}$ retorna um valor entre 0 e 1, em que 0 significa nenhuma reprodutibilidade e 1, a perfeita reprodutibilidade. A caracterização do $\mathrm{CCl}$ foi feita da seguinte forma: de 0,80-1,00, alta reprodutibilidade; de 0,60-0,79, moderada reprodutibilidade: $\mathrm{e} \leq 0,60$, baixa reprodutibilidade ${ }^{15}$. O erro também foi observado através do erro técnico da medida $\left(s=D \cdot P \cdot{ }_{\text {dif }} \div \sqrt{2}\right)$ e do coeficiente de variação (CV). Comparou-se os dados da eletromiografia dinâmica através da ANOVA com dois fatores para "momento" (testes versus reteste) versus "repetições" (uma a dez), e teste post-hoc de Tukey-HSD. Empregou-se o teste $t$ de Student para amostras pareadas na análise dos dados da EMG durante a CVM. Todos os testes estatísticos foram realizados no nível de significância de $p \leq 0,05$.

\section{RESULTADOS}

Os diagramas de dispersão do lado esquerdo das figuras 1 e 2 apresentam a relação entre o teste e o reteste na CVM e durante as 10 repetições, respectivamente, para a FM e a RMS.

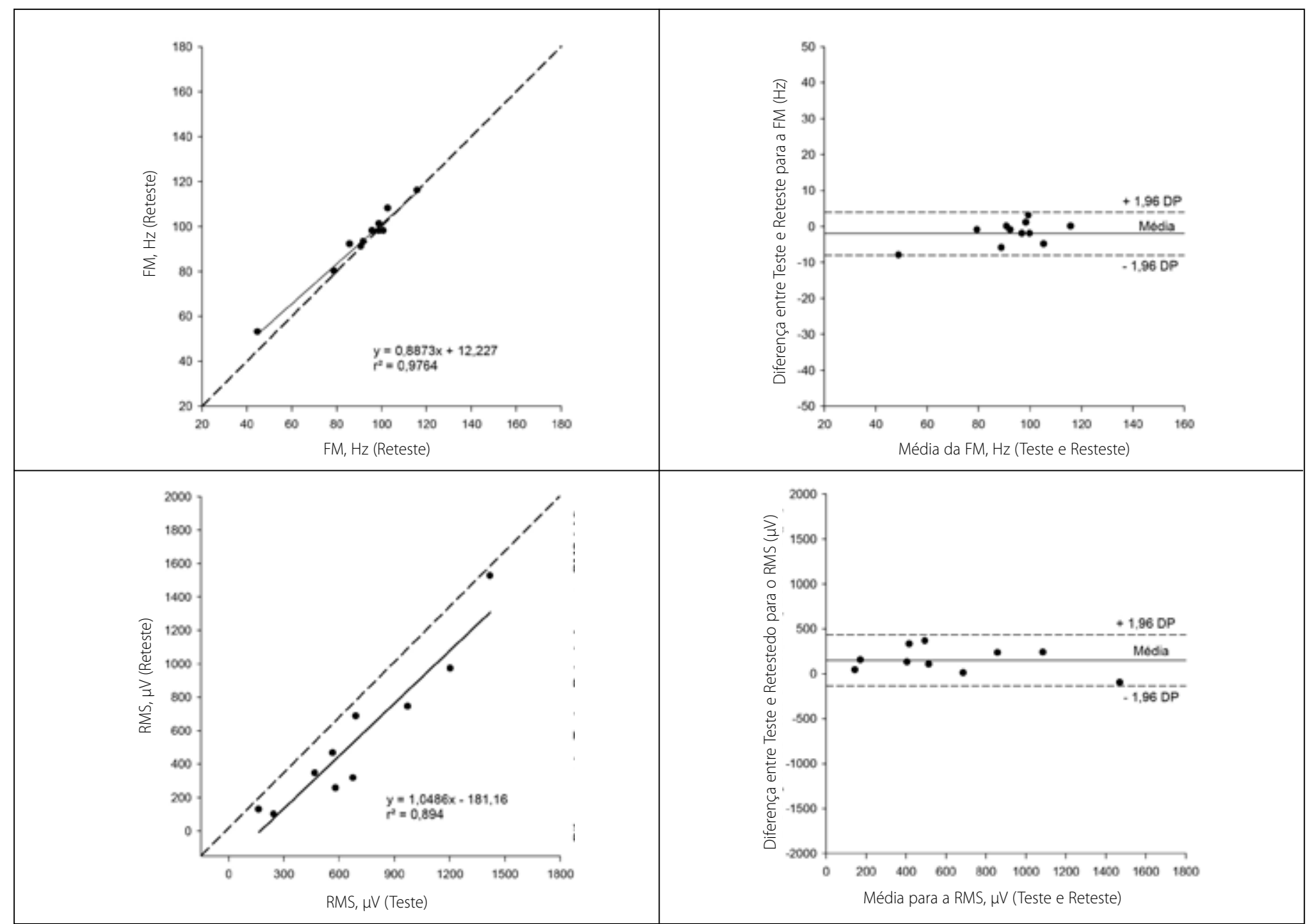

Figura 1. Teste de confiabilidade entre o teste e o reteste para a CVM e para as 10 repetições (figuras 1 e 2). Os gráficos à esquerda apresentam a relação entre o teste e o reteste através da linha de identidade (linha intermitente), linha de tendência (linha contínua) e equação de regressão com coeficiente de determinação (r2). Os gráficos à direita apresentam os limites de concordância de Bland-Altaman. As linhas escuras referem-se à média das diferenças e as linhas intermitentes, à variação para $\pm 1,96 \mathrm{DP}$. A figura 2 segue o mesmo formato. 


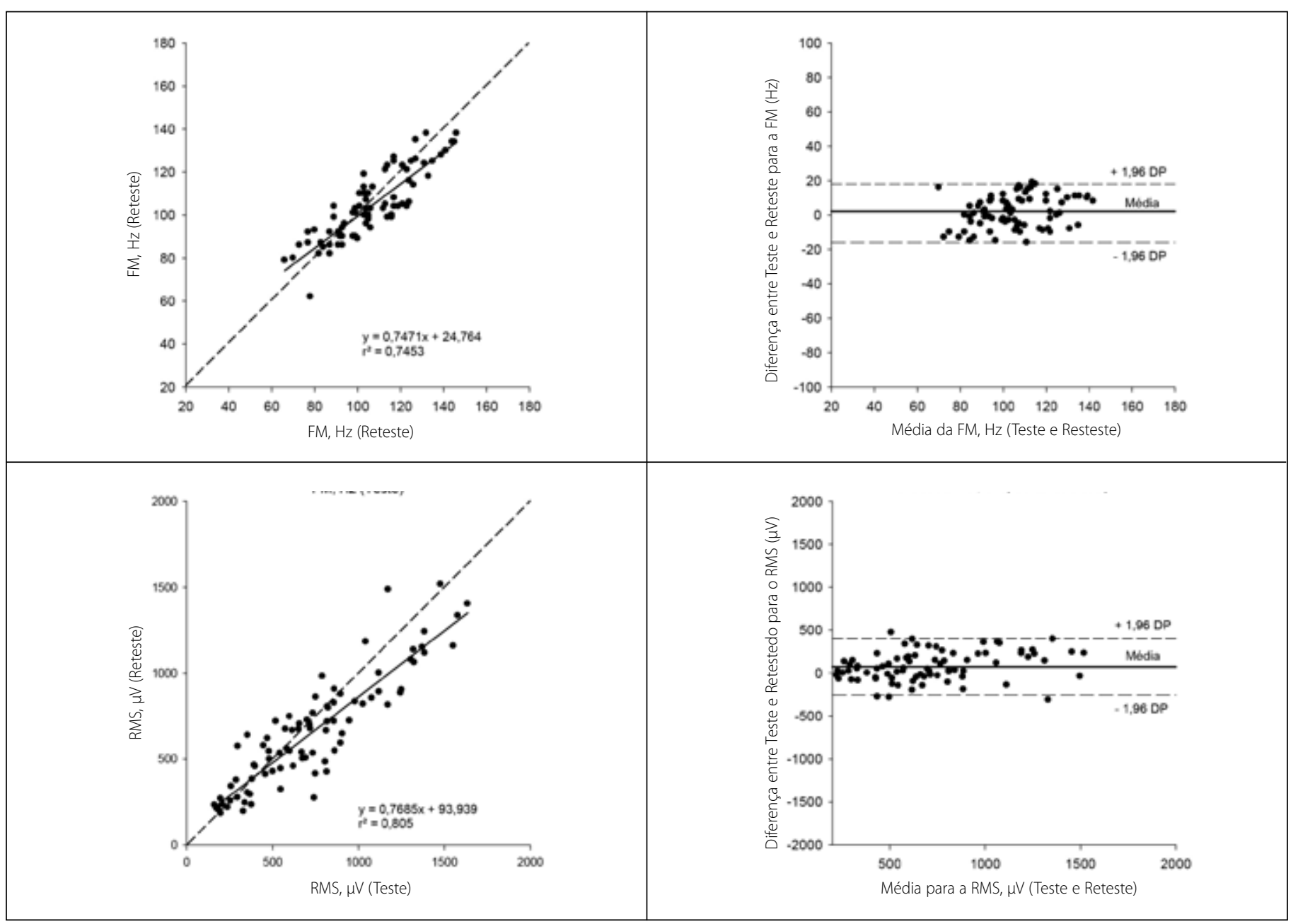

Figura 2. Teste de confiabilidade entre o teste e o reteste para as 10 repetições.

\section{Eletromiografia na contração voluntária máxima}

Observou-se uma FM média (EPM) de $92 \pm 6 \mathrm{~Hz}$ e no reteste de $94 \pm 5 \mathrm{~Hz}$, não sendo observada diferença significativa durante a CVM ( $p=0,798)$. Os limites de concordância observados foram de $-2 \pm 6 \mathrm{~Hz}$, $\mathrm{CCl}=0,982, \mathrm{CV}=2,5 \%$ e $\mathrm{s}=2 \mathrm{~Hz}$ entre o teste e o reteste, respectivamente, mostrando alta confiabilidade juntamente com um baixo erro típico. Root mean square - Observou-se um valor RMS médio no teste de $700 \pm 126 \mu \mathrm{V}$ e no reteste de $553 \pm 140 \mu \mathrm{V}$, respectivamente. Não foi observada diferença significativa durante a CVM $(p=0,444)$. Os limites de concordância foram de $147 \pm 284 \mu \mathrm{V}, \mathrm{CCl}=0,940, \mathrm{CV}=14,6 \%$, e s $=103 \mu \mathrm{V}$ entre o teste e o reteste.

\section{Eletromiografia durante 10 repetições dinâmicas}

Frequência mediana - Não foi observado, no confronto teste versus reteste, efeito principal $(p=0,434)$ ou interação significativa $(p=0,999)$. Foi observada diferença significativa apenas para repetições $(p=0,001)$. 0 teste de post-hoc mostrou diferença significativa (figura 3) entre a primeira, a nona e a décima repetição ( $p=0,025$ e $p=0,003$, respectivamente). A taxa de decrescimento da FM no teste foi de 14,7 e de 17,7\%, enquanto para o reteste foi de 18,7 e 24,1\%, quando comparamos a nona e a décima repetição com a primeira. Foram observados limites de concordância de $2 \pm 18 \mathrm{~Hz}, \mathrm{CCl}=0,90, \mathrm{CV}=6,2 \%$, e $\mathrm{s}=7 \mathrm{~Hz}$ entre o teste e o reteste. Root mean square - Não foi observado aumento significativo no valor da RMS comparando o começo com o final do teste (número de repetições, $p=0,815$ ). Não foi observado, também, efeito principal entre o teste versus o reteste $(p=0,191)$ ou interação significativa $(p=0,999)$. Os limites de concordância foram de $72,5 \pm 329 \mu \mathrm{V}, \mathrm{CCl}=0,89, \mathrm{CV}=16,3 \%$ e $s=119 \mu$ V entre o teste e o reteste.

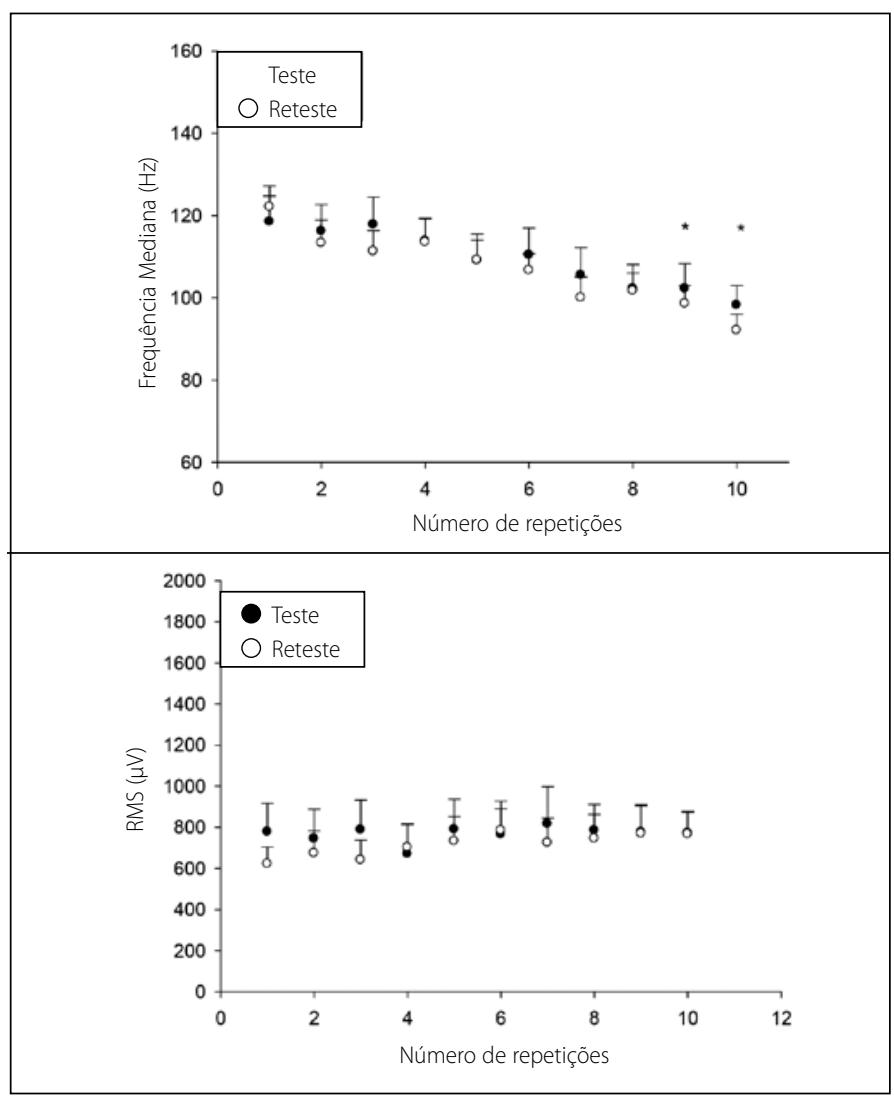

Figura 3. Resultados da frequência mediana (FM) à esquerda e da root mean square (RMS) à direita durante as 10 repetições. 


\section{DISCUSSÃO}

Um importante dado do presente estudo foi o alto CCI associado ao baixo erro típico da FM durante as contrações dinâmicas. A proposta do estudo foi determinar a reprodutibilidade das medidas da FM e RMS em exercícios dinâmicos e estáticos. Secundariamente, foi verificar a viabilidade da aplicação do sinal da EMG, através de sua análise no domínio da frequência como parâmetro para determinação e caracterização da fadiga neuromuscular do músculo bíceps braquial.

Durante exercícios dinâmicos e repetitivos muitos fatores podem contribuir para diminuição da reprodutibilidade da FM, demonstrada por um baixo CCl. Mesmo com todos os cuidados e precauções, pode ocorrer o deslizamento dos eletrodos na superfície da pele, afastamento dos tecidos musculares ativos embaixo do eletrodo, mudanças no comprimento das fibras musculares, mudanças no torque considerando toda a amplitude do movimento ${ }^{1,7,25}$. Além disso, o número de unidades motoras ativas muda rapidamente durante os exercícios dinâmicos ${ }^{14}$, o que pode implicar em não estacionaridade do espectro e, eventualmente, baixa validade da FM ${ }^{12}$. O esforço, entretanto, não apresentou diferença significativa entre o teste e o reteste, no presente estudo. Foi observada uma taxa de decréscimo similar em ambos os testes (figuras 1 e 2). As mudanças ocorreram a partir da nona contração em comparação com a primeira (figura 3), indicando diminuição na velocidade de condução do impulso neural como resposta aos efeitos da fadiga ${ }^{12,25}$.

$\mathrm{O}$ alto CCl da FM e da RMS pode ser devido à minuciosa padronização na execução do movimento de flexão do cotovelo. Dessa forma, aqueles fatores apresentaram influência constante a cada contração na execução da tarefa motora dinâmica. Foi observada, contudo, alta variabilidade no valor RMS (CV = 16,3\%). Estes valores são semelhantes aos relatados em estudos anteriores ${ }^{13}$. Uma boa reprodutibilidade também foi relatada em outros estudos, tanto na análise das contrações estáticas realizadas no mesmo dia quanto em diferentes dias ${ }^{13,15,26}$. A FM corresponde à velocidade de condução do impulso neural ${ }^{12}$. Representa as propriedades fisiológicas das unidades motoras ativas na execução das contrações dinâmicas. Consequentemente, a FM pode gerar informações mais detalhadas das propriedades estruturais e/ou funcionais dos músculos no desenvolvimento da fadiga.

O alto CCI para o RMS durante as contrações dinâmicas foi consistente com outros estudos que investigaram a amplitude do sinal em tarefas

\section{REFERÊNCIAS}

1. Miura $K$, Ishibashi $Y$, Tsuda $E$, Okamura $Y$, Otsuka $H$, Toh $S$. The effect of local and general fatigue on knee proprioception. Arthroscopy 2004;20:414-8.

2. Santos MCA, Semeghuini TA, Azevedo FM, Colugnati DB, Negrão Filho RF, Alves $N$, et al. Análise da fadiga muscular localizada em atletas e sedentários através de parâmetros de frequencia do sinal eletromiográfico. Rev Bras Med Esporte 2008;14:509-12

3. González-Izal M, Malanda A, Navarro-Amézqueta I, Gorostiaga EM, Mallor F, Ibañez J, et al. EMG spectral indices and muscle power fatigue during dynamic contractions. J Electromyogr Kinesiol 2010;20:233-40

4. James C, Scheuermann BW, Smith MP. Effects of two neuromuscular fatigue protocols on landing performance. J Electromyogr Kinesiol 2010;20:667-75

5. Bevilaqua-Grossi D, Felício LR, Simões R, Coqueiro KRC, Monteiro-Pedro V. Avaliação eletromiográfica dos músculos estabilizadores da patela durante exercício isométrico de agachamento em indivíduos com síndrome da dor femoropatelar. Rev Bras Med Esporte 2005;11:159-63.

6. Pizzato LM, Arakaki JC, Vasconcelos RA, Sposito GC, Oliveira AS, Paccola CJ, et al. Análise da freqüência mediana do sinal eletromiográfico de indivíduos com lesão do ligamento cruzado anterior em exercícios isométricos de cadeia cinética aberta e fechada. Rev Bras Med Esporte 2007;13:1-5.

7. Beck TW, Housh TJ, Johnson GO, Weir JP, Cramer JT, Coburn JW, et al. The effects of interelectrode distance on electromyographic amplitude and mean power frequency during isokinetic and isometric muscle actions of the biceps brachii. J Electromyogr Kinesiol 2005;15:482-95.

8. Gauche E, Couturier A, Lepers R, Michaut A, Rabita G, Hausswirth C. Neuromuscular fatigue following high versus low-intensity eccentric exercise of biceps brachii muscle. J Electromyogr Kinesiol 2009;19:e481-6.

9. Gabriel DA, Kamen G, Frost G. Neural adaptations to resistive exercise: mechanisms and recommendations for training practices. Sports Med 2006;36:133-49.

10. Malek MH, Coburn JW, Weir JP, BeckTW, Housh TJ. The effects of innervation zone on electromyographic amplitude and mean power frequency during incremental cycle ergometry. J Neurosci Methods 2006;155:126-33.

11. Allison GT, Fujiwara T. The relationship between EMG median frequency and low frequency band amplitude changes at different levels of muscle capacity. Clin Biomech (Bristol, Avon) 2002;17:464-9.

12. Merletti R, Lo Conte LR. Surface EMG signal processing during isometric contractions. J Electromyogr Kinesiol 1997;7:241-50.

13. Campy RM, Coelho AJ, and Pincivero DM. EMG-Torque Relationship and Reliability of the Medial and dinâmicas ${ }^{3,15}$. No presente estudo nenhuma mudança foi observada no valor RMS ao longo das 10 repetições. A execução de somente 10 repetições talvez não seja suficiente para alterar o padrão de recrutamento motor e provocar uma elevação significativa do RMS. Parece que houve apenas diminuição da frequência de disparo das $\mathrm{UMs}^{12}$. Provavelmente, as fibras recrutadas desde o começo do exercício apresentaram algum comprometimento na condução do impulso neural, observada pela diminuição da FM ${ }^{12}$. O último resultado reforça a viabilidade e a sensibilidade da FM como um bom parâmetro, com alta validade, para mensuração da fadiga neuromuscular em tarefas dinâmicas.

No presente estudo não foi observado aumento do valor RMS quando comparados os momentos inicial e final do teste. Nos exercícios máximos, ou próximos dessa intensidade, é necessário recrutar quase todas as UMs para gerar, ou manter, elevados níveis de força muscular ${ }^{12,27}$, causando aumento do valor RMS. O mecanismo fisiológico responsável pelo aumento da amplitude durante tarefas fatigantes não está claro. Contudo, é sugerido que o aumento na atividade elétrica ocorre devido ao progressivo recrutamento adicional de $U^{12,27}$, ou aumento da frequência de disparo das UMs já recrutadas ${ }^{7}$. Ocorre um aumento na amplitude da EMG em baixas bandas de frequência e uma redução relativa das bandas de altas frequências ${ }^{11}$.

\section{CONCLUSÃO}

Conclui-se que as medidas de FM e RMS apresentaram alta confiabilidade em protocolos de contrações sustentadas e dinâmicas envolvendo endurance muscular do músculo bíceps braquial. O baixo erro típico possibilita o uso destas medidas para investigação e monitoramento da progressão da fadiga neuromuscular através da EMG, tanto em contrações isométricas quanto dinâmicas.

\section{AGRADECIMENTOS}

Este estudo recebeu o apoio da Coordenação de Aperfeiçoamento de Pessoal de Nível Superior (CAPES), Fundação Carlos Chagas Filho de Amparo à Pesquisa do Estado do Rio de Janeiro (FAPERJ) e Conselho Nacional de Desenvolvimento Científico e Tecnológico (MCT/ CNPq).

Todos os autores declararam não haver qualquer potencial conflito de interesses referente a este artigo.

Lateral Hamstring Muscles. Med Sci Sports Exerc 2009;41:2064-7

14. Larsson B, Karlsson S, Eriksson M, Gerdle B. Test-retest reliability of EMG and peak torque during repetitive maximum concentric knee extensions. J Electromyogr Kinesiol 2003;13:281-7.

15. Callaghan MJ, McCarthy CJ, Oldham JA. The reliability of surface electromyography to assess quadriceps fatigue during multi joint tasks in healthy and painful knees. J Electromyogr Kinesiol 2009;19:172-80,

16. Loss JF, Candotti CT. Comparative study between two elbow flexion exercises using the estimated resultant muscle force. Rev Bras Fisioter 2008;12:502-10.

17. Rocha JúniorVA, Gentil G, Oliveira E, Carmo J. Comparação entre a atividade EMG do peitoral maior, deltóide anterior e tríceps braquial durante os exercícios supino reto e crucifixo. Rev Bras Med Esporte 2007;13:51-4.

18. Bonato P. Roy SH, Knaflitz M. De Luca CI Time-frequency parameters of the surface myoelectric signal for assessing muscle fatigue during cyclic dynamic contractions. IEEE Trans Biomed Eng 2001;48:745-53.

19. Farina D. Interpretation of the surface electromyogram in dynamic contractions. Exerc Sport Sci Rev 2006;34:121-7.

20. Tank FF, Silva GT, Oliveira CG, Garcia MAC. Influência da distância intereletrodos e da cadência de movimento no domínio da frequência do sinal de EMG de superfície. Rev Bras Med Esporte 2009;15:272-6.

21. Gomes PSC, Meirelles CM, Leite SP, Montenegro CAB. Confiabilidade da medida de espessuras musculares pela ultrassonografia. Rev Bras Med Esporte 2010;16:41-5.

22. Larsson B, Månsson B, Karlberg C, Syvertsson P, Elert J, Gerdle B. Reproducibility of surface EMG variables and peak torque during three sets of ten dynamic contractions. J Electromyogr Kinesiol 1999;9:351-7.

23. Jackson AS, Pollock ML. Generalized equations for predicting body density of men. Br J Nutr 2004;91:161-8.

24. Hermens HJ, Freriks B, Merletti R, Stegeman D, Blok J, Rau G, Disselhorst-Klug C, Hägg G, SENIAM 8: European Recommendations for Surface Electromyography (Roessingh Research and Development, Enschede), 1999.

25. Masuda K, Masuda T, Sadoyama T, Inaki M, Katsuta S. Changes in surface EMG parameters during static and dynamic fatiguing contractions. J Electromyogr Kinesiol 1999;9:39-46.

26. Kellis $E$, Katis A. Reliability of EMG power-spectrum and amplitude of the semitendinous and biceps femoris muscles during ramp isometric contractions. J Electromyogr Kinesiol 2008;18:351-8.

27. Baudry S, Rudroff T, Pierpoint LA, Enoka RM. Load type influences motor unit recruitment in biceps brachii during a sustained contraction. J Neurophysiol 2009;102:1725-35. 